\title{
A TENTH-CENTURY FRAGMENT OF TERTULLIAN'S APOLOGY.
}

IN view of the slender ancient testimony to the text of Tertullian's Apologv, it seems worth while to report the readings of a tenthcentury MS of chapters 38,39 , and part of 40 , especially as its text is closely related to that of the important Fulda MS which is now lost. The excellent manuscript catalogue of the Rheinau collection, now in the Kantons-Bibliothek in Zürich, has never been printed, and it is probably on this account that the fragment has hitherto escaped notice. MS xcv (saec. x) is a collection of passages from various authors which interested the compiler, something after the fashion of the 'Collectaneum' of Sedulius Scottus at Cues on the Mosel. ${ }^{1}$ Among these are to be found the De XII Abusiuis Sacculi, which is sometimes attributed to Cyprian, and on pp. 175-1 84 Tertullian's Apology, chaps. 38-40 (down to tantos ad unum $=$ Oehler I (Lips. 1853) p. 267,4). I here give a collation of the extract with Oehler's text.

\section{Oehler.}

nec

licitas

timeri solet

constat

quae res

concilia curias

contiones

inquietaret

quaestu

coepissent

homines

nobis

gloriae

unam

aeque

renuntiamus

eorum

est

dictu
Rheinaug. etc. ( $\lambda=$ Fulda MS).

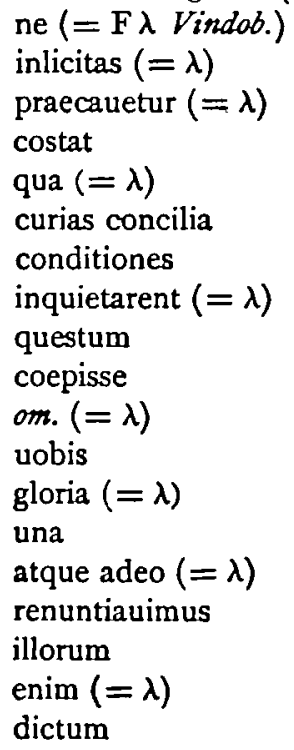

dictum

1 For which see S. Hellmann's Sedulius Scottus (Munchen, 1906). 
Oehler.

bost uanitate nihil

nouisse

reprobamus

Sed licuit Epicu-

reis aliquam de-

cernere uolupta-

tis ueritatem id

est animi aequita-

tem et ampla

negotia Christianae

ut qui

ostendam

coetum

congregationem

orantes

ministris

pascimus

praeceptorum nihilominus

inculcationibus

futuri iudicii

honoraria

conpellitur

confert

nam inde

ingratiis

ac puellis re

destitutis

senibus

sectae

nobis inurit

et ut (=A B G $\lambda$ )

enim
Rheinaug. etc.

bost uanitate habet

licuit epicureis aliam

decernere uoluptatis ueri-

tatem, id est anima (animae $\lambda$ ) equi-

tatem. In $(=\lambda)$

nouissime $(=\lambda)$

probamus

om. $(=\lambda)$ quo minus $(=\lambda)$

ostendam si etiam

reuelauerim ueri-

tatem (cf. $\lambda$ uer. reuel.)

coetu $(=\lambda)$

congregationem facimus $(=\lambda)$

om. $(=\lambda)$

ministeriis $(=\lambda)$

poscimus

nihilominus praeceptorum

in conpulsationibus $(=\lambda)$

iudicii futuri

oneraria $(=(\lambda))$

conpellitus

confret $^{1}$

quippe $(=\lambda)$

ingratis $(=G \lambda)$

om. $(=\lambda)$

destitus

senibus iam

otiosis $(=\lambda)$

sectae conflic-

tantur (cf. $\lambda$ conflictatur)

uobis inurit

et $(=\mathrm{D} \mathrm{E})$

enim sunt

1 This spelling 1 have also seen in Clm. 6312 (saec. ix) of Py. -Aug. Quaest. 
Oehler.

alterutrum

erunt

fratres nos vo-

camus

opinor

quam quod

At ( $=\lambda$ etc.)

quanto

patrem deum

spiritum biberint sanctitatis

exclamat

ex

loco

maiorum et sapi-

entissimorum

quam

donauerant

lenones

philosophus

conviolatur

coenulus

morituri (alt.)

Saliis

Herculanarum

polluctorum

Apaturiis

Dionysiis

delectus

indicitur

Sarapiacae

sparteoli

de solo

vocatur quod

refrigerio

parasiti

saginandi

qua

est convivii

ut qui (pr.)

deum sibi

dominum
Rheinaug. etc.

alterutro

om. $(=\lambda)$

fratrum appel-

latione censemur ( $\lambda$, sed hic censemus)

opinior

quam cum

om.

quanto nunc (cf. $\lambda$ quando nunc)

deum patrem

sanctitatis spiritum biberunt (quoad biberunt $\lambda$ etc.)

exclamant

om. (?)

solo $(=\lambda)$

malorum et suorum

sapientiorum (cf. $\lambda$ sapientiorum suorum)

quas

donauerunt

leno est $(=\lambda$ etc.)

philosopus

conuiuatur $(=\lambda)$

caenula

moriantur $(=\lambda)$

si aliis $(=\lambda$ etc.)

herculanorum

polincto lucitorum $(=\lambda)$

apparaturis

aconisi

dilectus

inducitur

se arapia ae (cf. $\lambda$ serapiae)

spartioli

doloso ( $\lambda$ habet de loco)

uocatum quō ( $\lambda$ uocatum quo)

refrigiorio

parasti

sagenandi

quia

conuiuii est

ut $(=\lambda)$

sibi deum

$\operatorname{deum}(=F \lambda)$ 
Oehler.

sanctis

provocatur in medi-

um deo canere

in eruptiones

ut

damnanda

om.

de ea queritur

quo

cuius

neminem laedentes

accommodandum

qui adversum

sane

omnis publicae cla-

dis omnis popula-

ris incommodi

arva

stetit

adclamatur

diuinis

de deo canere pro-

uocatur in medio (cf. $\lambda$ de deo)

ad inreptiones ( $\lambda$ in inceptiones)

et

sane damnanda

si non dissimilis

damnandis $(=\lambda)$

deaquaeritur

quō

ciuius

om.

adcommodandum

quid aduersus

plane

omnis popularis

omnis publicae

cladis incommodi

in primordio tem-

porum ( $\lambda$ habet

in primordio temporum)

rura $(=\lambda)$

non stetit

om. (= aliquot edd. : $\lambda$ habet inclamant).

The close relationship between the Zürich extract and the Fulda MS is at once evident. ${ }^{2}$ The accuracy with which the old scholars collated the latter is incidentally illustrated. Some readings given above are manifestly wrong, but they may help in tracing the date and character of this special form of text. Other readings are mere variations of order. The remainder seem worthy of consideration.

\section{Alex. Souter.}

1 Compare the case of the Fulda (formerly Weingarten) and Rheinau MSS of Sedulius Scottus. Hellmann (op. cit. p. Igo ff) has shewn that they are both copies of the same lost MS. 